American Journal of Infectious Diseases 2 (3): 187-192, 2006

ISSN 1553-6203

(c) 2006 Science Publications

\title{
Molecular Mechanisms for Altered Glucose Homeostasis in HIV Infection
}

\author{
Paul W Hruz \\ Department of Pediatrics, Department of Cell Biology and Physiology \\ Washington University School of Medicine, St. Louis, MO 63110
}

\begin{abstract}
A complete understanding of the molecular mechanisms leading to HIV-associated insulin resistance remains elusive. Complex interrelationships between genetic predisposition, disease-related body changes and multidrug therapy all contribute to alterations in glucose homeostasis. These abnormalities can be differentiated between acute and reversible changes directly induced by HAART medications and more chronic and less reversible changes due to the development of lipodystrophy and hyperlipidemia. Implicated pathways include changes in adipokine secretion, insulin signaling, lipid homeostasis and disease-related increases in inflammatory mediators. The insulin responsive facilitative glucose transporter GLUT4 is the first molecule to have been identified as a direct target of HIV protease inhibitors. Efforts to elucidate the mechanisms directly responsible for the evolution of insulin resistance during HIV infection and therapy will be greatly assisted by the further identification and characterization of direct molecular targets amenable to pharmacologic therapy and/or the development of newer antiretroviral agents that do not adversely affect these target proteins.
\end{abstract}

Key words: HIV infection, molecular mechanism, homeostasis

\section{INTRODUCTION}

Among the many metabolic perturbations that occur as a result of HIV infection and its treatment, alterations in normal glucose homeostasis remain a particularly prevalent and alarming clinical change in affected patients ${ }^{[1]}$. Much of this concern is due to the recognition of the long-term complications of insulin resistance and hyperglycemia as understood in the context of the growing worldwide epidemic of type 2 diabetes mellitus ${ }^{[2]}$. As a result, there remains considerable interest in elucidating the molecular mechanisms responsible for disordered glucoregulation during highly active antiretroviral therapy (HAART). Goals of current research efforts in this area are many. They include the identification of ways to predict those patients at greatest risk for developing diabetes, the modification of existing antiviral regimens to minimize this risk and the tailoring of diabetes treatment strategies toward the underlying genetic and/or molecular defects present in these patients. Ultimately, the goal is to develop newer antiretroviral agents that maintain their clinical efficacy in preventing HIV replication without causing their deleterious metabolic effects. While progress has been made in defining the epidemiology of insulin resistance in the HIV infected patient and a clearer appreciation of the relative contributions of each of the components of HAART is starting to emerge ${ }^{[3]}$, significant gaps remain in our understanding of the molecular basis of insulin resistance in this setting.
In attempting to identify the mediators of impaired glucose tolerance during HIV infection, it is important to first acknowledge both the similarities and differences in this clinical setting compared to the general population. The genetic and environmental factors present in HIV negative individuals co-exist in HIV infected patients and can independently contribute to altered glucose homeostasis. While poor nutrition and wasting were prevalent manifestations of HIV infection in the pre-HAART $\mathrm{era}^{[4]}$, the successful containment of viral replication and immune reconstitution have led to the re-emergence of nutritional excess as a contributing problem ${ }^{[5]}$. This generalized fat accumulation, distinct from the lipodystrophic changes found in the HIV dysmetabolic syndrome, is "fueled" by the consumption of the same high fat diet and sedentary lifestyle characteristic of western societies. Thus, not all of the increased visceral adiposity observed may be directly related to HIV therapies. Type 2 diabetes is also recognized to be a polygenetic disease such that relatively small defects in multiple mediators of normal insulin signaling pathways can collectively lead to the overt manifestation of impaired glucose tolerance ${ }^{[6]}$. In this context, further perturbations in glucoregulation induced by HIV infection may lead to clinical changes that would not otherwise be apparent. Therefore, while it is important to investigate specific mechanisms for insulin resistance in HIV infection, the environmental and genetic setting in which this occurs cannot be ignored. This is particularly important in attempting to 
extrapolate study results to potential effects on insulin sensitivity in the developing world.

With this in mind, it is apparent that there are multiple factors related to HIV infection and its treatment that directly contribute to the development of insulin resistance. Since the goal is to define precise etiologic mechanisms, this review will focus upon the putative and established molecular targets influencing normal glucose metabolism which are altered during HAART. Any alteration in the expression and/or activity of each of these proteins has the significant potential for causing multiple downstream changes in affected pathways. Compensatory changes will also frequently occur over time, particularly when critical pathways are perturbed. Therefore, an emphasis will be made on acute changes occurring within the timeframe of minutes to hours rather than more chronic changes occurring over weeks to months. While the significant metabolic changes occurring under chronic conditions may overshadow many of these acute changes, it is likely that the greatest chance for establishing the most proximate effects of antiretroviral therapy on insulin sensitivity is to be found by examining these earlier timepoints.

While the fundamental aspects of normal glucoregulation have been studied and understood for decades, knowledge in this area continues to grow at an incredible pace within recent years ${ }^{[7]}$. Notable is a growing understanding of the role of the adipocyte as an endocrine organ ${ }^{[8]}$. We are only now starting to identify and characterize the normal regulation and function of adipokines such as leptin, adiponectin and resistin ${ }^{[9]}$. In addition, the molecules involved in the distal portions of the insulin signaling pathway are still being identified and the intricate relationships between many seemingly divergent metabolic pathways are beginning to be deciphered ${ }^{[10]}$. Continued progress in these areas will certainly aid efforts to understand the molecular mechanisms responsible for altered glucose homeostasis during HAART.

Since regulation of glucose flux is dependent upon the coordinated action of many tissues, particular attention should be made to the effects of changes in candidate molecular targets in each of these tissues. While defects in peripheral glucose disposal, particularly skeletal muscle, appear to be a predominate change observed during HAART ${ }^{[11]}$, key insights into etiologic mechanisms and potential therapeutic solutions can be obtained by also considering effects on endogenous glucose production from the liver, insulin secretion from the $\beta$-cell and the endocrine function of adipocytes.

Inflammatory cytokines: Circulating cytokine levels, particularly TNF- $\alpha$ and IL-6, are frequently elevated in HIV positive patients ${ }^{[12]}$. Furthermore, co-morbid conditions associated with HIV, such as hepatitis $\mathrm{C}$ infection $^{[13]}$ also independently contribute to elevated
TNF- $\alpha$ levels. It is well established that such elevations in TNF- $\alpha$ can cause significant impairment of insulin action $^{[14]}$. The molecular mechanism(s) by which elevations in TNF- $\alpha$ and other inflammatory cytokines lead to insulin resistance is incompletely understood ${ }^{[15]}$. However, these cytokines can influence both normal suppression of hepatic glucose production and insulin stimulated glucose uptake. In addition, TNF- $\alpha$ can indirectly influence peripheral insulin sensitivity by stimulating triglyceride and free fatty acid production in the liver.

Initial evaluations of insulin sensitivity in HIVinfected patients, particularly in the pre-HAART era, suggested that virus infection itself was not sufficient to change insulin sensitivity ${ }^{[16]}$. In treatment-naïve patients with wasting, insulin sensitivity was actually increased $^{[4]}$. However, reflecting the adipocyte's ability to secrete inflammatory cytokines, there appears to be a close link between hypersecretion of TNF- $\alpha$ and visceral adiposity in the more generalized HIV independent metabolic syndrome ${ }^{[17]}$. Thus, the additive effect of changes in body fat observed during HAART, with increased cytokine secretion from visceral adipocytes and elevated cytokine production from infection may lead to sufficient changes in insulin sensitivity to produce clinical symptoms ${ }^{[12]}$. However, the potential magnitude of this contribution relative to the effects of antiretroviral therapy is not clear. It was not until the widespread introduction of HIV protease inhibitors (PIs) in the late 1990's that the dramatic increase in reported cases of altered glucose homeostasis was recognized ${ }^{[18]}$. Since PIs appear to play a primary role in the development of HAART associated insulin resistance, primary consideration of the cellular effects of these drugs will be considered in discussing direct molecular targets.

GLUT4: The elucidation of changes in glucose transport in the induction of peripheral insulin resistance is perhaps one of the clearest examples of the utility of focusing efforts on the identification of direct targets of antiretroviral drugs. Recent progress in this area has allowed the establishment of the insulin responsive facilitative glucose transporter GLUT4 as a proximate cause of altered insulin sensitivity. To date, this molecule remains the only protein to have been definitively identified as a direct target of antiretroviral therapy. While changes in the expression and/or function of other potential target molecules are likely to contribute to significant changes in whole-body glucose homeostasis, the establishment of these proteins as direct mediators, rather than secondary effectors, is still largely lacking. Though it remains entirely possible, if not likely, that additional drug targets will be identified, the experience with GLUT4 can serve as an excellent example of the type of research that is still needed to fully characterize, prevent and/or treat HIV associated 
insulin resistance. In addition to the insights that can be gained regarding the direct cellular effects of PI therapy, such research efforts are also likely to provide valuable new clues to the regulatory mechanisms influencing glucose levels in normal and HIVindependent diseased states.

The initial observation that established GLUT4 as a candidate molecular target of PIs was the finding that indinavir acutely (i.e. within minutes) inhibits insulinstimulated glucose uptake into 3T3-L1 adipocytes ${ }^{[19]}$. Furthermore, it was found that this inhibition occurs without any detectable changes in GLUT4 translocation to the plasma membrane and can be induced with drug addition following maximal insulin stimulation. These results clearly differ from later reports that have shown that under chronic conditions, PIs can affect insulin signaling ${ }^{[20]}$. Importantly, inhibition of glucose uptake is also observed in a heterologous GLUT4 expression system that is not insulin responsive ${ }^{[19]}$. While the drug concentrations used in initial experiments far exceeded the PI levels typically achieved during HAART, subsequent studies in primary rat adipocytes confirmed that the acute effect on GLUT4 activity is pharmacologically relevant ${ }^{[21]}$.

To link these in vitro findings to effects on whole body glucose homeostasis, hyperinsulinemic euglycemic clamps, the gold standard test for peripheral insulin sensitivity, were performed on healthy rodents following acute exposure to indinavir ${ }^{[22]}$. As predicted, significant insulin resistance was observed immediately following the administration of the PI and full insulin sensitivity could be restored following drug removal. Supporting a direct link between these laboratory findings and clinically relevant drug effects, it was found that a single dose of indinavir to HIV negative healthy volunteers was sufficient to induce peripheral insulin resistance ${ }^{[23]}$. Importantly, these changes were observed without any of the other associated features of the HIV dysmetabolic syndrome.

While these studies have established that GLUT4 inhibition is a primary mechanism by which indinavir acutely induces insulin resistance, attempts to extrapolate these findings to other PIs has been less clear. Clinically, indinavir appears to be the most potent of the PIs in inducing significant changes in insulin sensitivity. Other PIs, such as amprenavir, do not appear to be as effective in causing acute insulin resistance $^{[24]}$. Thus, it has been proposed that GLUT4 inhibition represents a drug specific effect rather than a general effect of all PIs. This contrasts with the observation that GLUT4 inhibition in vitro can be produced with nearly all of the currently available PIs ${ }^{[19]}$. Since the $K_{i} s$ for GLUT4 inhibition for the PIs are all in the low micromolar range (unpublished observations), it is possible that the apparent discrepancy between the observed in vitro and in vivo effects of the PIs is primarily related to differences in the peak levels of bioavailable drug achieved during clinical use. In general, PIs are highly hydrophobic compounds with a significant degree of protein binding in serum. Since indinavir has the least amount of protein binding, it is not surprising that this PI is the most potent inducer of insulin resistance in vivo.

A notable exception to the above generalization is the newest PI atazanavir, which has not been found to be associated with acute insulin resistance in vivo ${ }^{[25]}$. Out of all of the available PIs, atazanavir is the only drug to have no observable effects on in vitro glucose transport activity ${ }^{[26]}$. In consideration of the GLUT4 inhibitory properties of candidate PIs together with protein binding capacity and the necessary drug levels achieved during clinical use, the above data indicate that a simple in vitro assay for acute effects on insulin stimulated glucose uptake can reasonably predict the potential to induce acute insulin resistance in treated patients.

Nevertheless, while potentially valuable in large screening trials of drugs in development, such information does not provide a useful method to design and develop newer generations of PIs, one of the primary goals of research in this area. However, with identification of a relevant molecular target, it now becomes possible to investigate the precise structural basis for the adverse metabolic effect in relation to changes in the desired beneficial effect in controlling HIV replication. Such structurally based studies with GLUT4 are now in progress. Recent work has established that the core peptidomimetic structure within PIs is necessary for GLUT4 inhibition and affinity labeling studies have confirmed that the adverse effect on glucose uptake is due to direct binding of PIs to this transporter ${ }^{[27]}$.

GLUT2: In addition to inducing peripheral insulin resistance, PIs have also been found to contribute to impaired insulin release from $\beta$-cells, which increases the risk of patients to develop overt diabetes mellitus ${ }^{[11]}$. Again, in vitro studies have provided insights into the molecular mechanism(s) for impaired glucose stimulated insulin release from these cells. While the direct molecular targets for PIs in these cells has not yet been established, defective glucose transport and/or metabolism has been implicated ${ }^{[28]}$. Thus, it is possible that the effects on $\beta$-cell function are due in part to acute changes in the function of the related glucose transporter(s) present in this tissue, namely GLUT2. This molecule, which is expressed in both the $\beta$-cell and liver, has a $\mathrm{K}_{\mathrm{m}}$ for glucose which is well within the physiologic range of serum glucose levels. For this reason, this protein has been proposed to function as a glucose sensor in these tissues. In addition to effects on insulin secretion, PIs have been found to have acute effects on glycogen synthesis in cultured hepatocytes ${ }^{[29]}$. It remains to be determined whether altered glucose transport is contributing to this effect. 
Similar to the differences in acute versus chronic effects on peripheral glucose disposal, the effects of PIs on $\beta$-cell function can be strongly influenced by the timeframe in which studies are performed. In contrast to the acute effects of PIs on glucose-stimulated insulin release, more chronic exposure to PIs appears to alter insulin signaling with clear differences between PIs ${ }^{[30]}$. Whether this is the result of direct alterations on other protein targets or secondary changes following glucose transport inhibition remains to be determined.

Free fatty acids: Since increased incidence of insulin resistance during HAART was first identified in conjunction with other metabolic changes, namely lipodystrophy and hyperlipidemia, it was initially believed that decreased insulin sensitivity was secondary to these chronic changes. However, it is now clear that PIs have direct effects on insulin sensitivity that are independent from the lipodystrophic and/or hyperlipedemic changes ${ }^{[31]}$. Increases in free fatty acid levels, whether directly or indirectly resulting from PI use, can contribute to altered glucose homeostasis, both through toxic effects on peripheral insulin signaling ${ }^{[32]}$ and impaired $\beta$-cell responsiveness ${ }^{[33]}$.

Recent in vitro studies have linked the use of PIs to alterations in the expression and/or processing of the sterol response element binding protein SREBP$1 c^{[20,34]}$. This protein normally regulates the expression of several genes important in fatty acid synthesis. While speculation has been made regarding the role of altered SREBP processing in the evolution of lipodystrophic changes through effects on adipocytes differentiation, this molecule may also have more direct influences on insulin sensitivity through acute changes in intracellular lipid levels. The relative rapidity in which changes can be observed in vitro makes this molecule attractive as a direct target of PIs. Nevertheless, it has yet to be established whether PIs have any direct association with the SREBP or the molecules affecting its processing. Since SREBP activity is strongly correlated with nutritional status, it is possible that these changes reflect changes in cellular energy, perhaps secondary to effects on glucose flux. Attempts to more carefully delineate whether SREBP-1c changes are primary versus secondary cellular effects will greatly facilitate efforts to determine the role of this protein in the development of insulin resistance.

Leptin: Increased interest in the potential role of altered adipokine secretion during HAART has coincided with the growing recognition of the role of these hormones in normal glucose homeostasis ${ }^{[35]}$. Leptin, one of the earliest of the adipocyte-secreted hormones to be identified, has been characterized as a regulator of total body fat mass via central effects on appetite in the hypothalamus. This hormone also appears to have significant effects on peripheral insulin sensitivity. The administration of leptin to patients with congenital generalized lipodystrophy leads to a rapid and dramatic improvement in peripheral insulin sensitivity ${ }^{[36]}$. Exogenous leptin has also been shown to ameliorate hyperlipidemic and lipodystrophic changes in PI-treated mice ${ }^{[37]}$.

Given the close association between adipocyte mass and leptin levels, it is not surprising that leptin levels are correlated with lipodystrophic changes in HIV infected patients ${ }^{[38]}$. However, leptin levels do not appear to correlate with insulin resistance in this lipodystrophic patient population. Despite the likelihood that changes in leptin levels are secondary to changes in body fat, it remains possible that more direct effects of PIs on leptin expression and/or secretion also may occur on a more acute timescale. Insulin signaling is known to directly stimulate leptin secretion ${ }^{[39]}$. Furthermore, inhibition of glucose transport with either cytochalasin $\mathrm{b}$ and/or phloretin can reduce leptin secretion from cultured adipocytes ${ }^{[40]}$. Thus, it is again possible that the direct inhibition of GLUT4 activity induced by PIs secondarily leads to changes in adipocyte leptin expression, even before lipodystrophic changes occur.

Adiponectin: The novel adipocyte-secreted hormone adiponectin also is known to significantly affect whole body glucose homeostasis, both by enhancing hepatic insulin action and peripheral insulin sensitivity ${ }^{[41]}$. The correlative link between reductions in adiponectin levels and HIV-associated lipodystrophy has established this hormone as a potential mediator of insulin resistance in affected patients ${ }^{[42,43]}$. While it is possible that these changes are again merely secondary to changes in body fat, recent studies have shown that PIs can produce relatively acute dose-related reductions adiponectin levels in vitro and in vivo ${ }^{[44]}$. Within this mouse model system, ritonavir and adiponectin did not significantly alter indirect measures of insulin sensitivity. However, exogenous adiponectin administration was found to reverse the hyperlipidemia induced by ritonavir treatment.

Complicating the interpretation of the significance of these findings, a recent study has reported that treatment of healthy HIV negative subjects with PIs increases, rather than decreases adiponectin levels ${ }^{[45]}$. Given the apparent complexity of glucose regulatory pathways, it is possible that with longer periods of drug exposure, compensatory mechanisms are stimulated, masking or abrogating changes directly induced by the drugs. Further studies are still needed to explore the direct molecular mechanism by which PIs alter adiponectin expression and/or secretion.

\section{CONCLUSION}

The problem of identifying and characterizing the molecular mechanisms for altered glucose homeostasis during HIV infection has proven to be a challenging 
task for both basic scientists and clinicians alike. Due to the large number of clinical variables involved and the complexity of the signaling pathways that participate in glucoregulation, attempts to address the problem in clinical trials have thus far been met with only limited success. While such studies have generated a large (and growing) list of potential mediators, the investigation of acute changes in the expression and/or function of these molecules in vitro and within controlled animal model systems has the potential to provide valuable mechanistic information to guide the development of safer HIV treatment regimens. A better knowledge and appreciation for the most proximate changes in the molecules controlling insulin sensitivity resulting from HIV infection and treatment will provide a crucial framework for the further dissection of the complex relationships between affected pathways.

\section{ACKNOWLEDGEMENT}

Financial support by National Institute of Allergy and Infectious Diseases (AI49747); National Institute of Diabetes and Digestive and Kidney Diseases (DK64572) are acknowledged.

\section{REFERENCES}

1. Koutkia, P. and S. Grinspoon, 2004. HIVAssociated lipodystrophy: Pathogenesis, prognosis, treatment and controversies. Annu. Rev. Med., 55: 303-317.

2. Zimmet, P., K.G.M.M. Alberti and J. Shaw, 2001. Global and societal implications of the diabetes epidemic. Nature, 414: 782-787.

3. Hadigan, C., J.B. Meigs and C. Corcoran et al., 2001. Metabolic abnormalities and cardiovascular disease risk factors in adults with human immunodeficiency virus infection and lipodystrophy. Clin. Infect. Dis., 32: 130-139.

4. Hommes, M.J., J.A. Romijn and E. Endert et al., 1991. Insulin sensitivity and insulin clearance in human immunodeficiency virus-infected men. Metabolism, 40: 651-656.

5. Kruzich, L.A., G.S. Marquis and C.M. Wilson et al., 2004. HIV-infected US youth are at high risk of obesity and poor diet quality: A challenge for improving short- and long-term health outcomes. J. Am. Diet. Assoc., 104: 1554-1560.

6. Florez, J.C., J. Hirschhorn and D. Altshuler, 2003. The inherited basis of diabetes mellitus: Implications for the genetic analysis of complex traits. Annu. Rev. Genomics. Hum. Genet., 4: 257291.

7. DeFronzo, R.A., 2004. Pathogenesis of type 2 diabetes mellitus. Med. Clin. North Am., 88: 787835 .
8. Kershaw, E.E. and J.S. Flier, 2004. Adipose tissue as an endocrine organ. J. Clin. Endocrinol. Metab., 89: 2548-2556.

9. Meier, U. and A.M. Gressner, 2004. Endocrine regulation of energy metabolism: Review of pathobiochemical and clinical chemical aspects of leptin, ghrelin, adiponectin and resistin. Clin. Chem., 50: 1511-1525.

10. Saltiel, A.R. and C.R. Kahn, 2001. Insulin signalling and the regulation of glucose and lipid metabolism. Nature, 414: 799-806.

11. Woerle, H.J., R.R. Mariuz and C. Meyer et al., 2003. Mechanisms for the deterioration in glucose tolerance associated with HIV protease inhibitor regimens. Diabetes, 52: 918-925.

12. Mynarcik, D.C., M.A. McNurlan and R.T. Steigbigel et al., 2000. Association of severe insulin resistance with both loss of limb fat and elevated serum tumor necrosis factor receptor levels in HIV lipodystrophy. J. Acquir. Immune. Defic. Syndr., 25: 312-321.

13. Mohsen, A.H., P., Easterbrook and C.B. Taylor et al., 2002. Hepatitis C and HIV-1 coinfection. (Review). Gut, 51: 601-608.

14. Borst, S.E., 2004. The role of TNF-alpha in insulin resistance. Endocrine, 23: 177-182.

15. Hotamisligil, G.S., 2003. Inflammatory pathways and insulin action. Intl. J. Obes. Relat. Metab. Disord., 27 Suppl 3: S53-55.

16. Hellerstein, M.K., C. Grunfeld and K. Wu et al., 1993. Increased de novo hepatic lipogenesis in human immunodeficiency virus infection. J. Clin. Endocrinol. Metab., 76: 559-565.

17. Katsuki, A., Y. Sumida and S. Murashima et al., 1998. Serum levels of tumor necrosis factor\{alpha\} are increased in obese patients with noninsulin-dependent diabetes mellitus. J. Clin. Endocrinol. Metab., 83: 859-862.

18. Walli, R., O. Herfort and G.M. Michl et al., 1998. Treatment with protease inhibitors associated with peripheral insulin resistance and impaired oral glucose tolerance in HIV-1-infected patients. AIDS, 12: F167-173.

19. Murata, H., P.W. Hruz and M. Mueckler, 2000. The mechanism of insulin resistance caused by HIV protease inhibitor therapy. J. Biol. Chem., 275: 20251-20254.

20. Caron, M., M. Auclair and C. Vigouroux, et al., 2001. The HIV protease inhibitor indinavir impairs sterol regulatory element-binding protein-1 intranuclear localization, inhibits preadipocyte differentiation and induces insulin resistance. Diabetes, 50: 1378-1388.

21. Murata, H., P.W. Hruz and M. Mueckler, 2002. Indinavir inhibits the glucose transporter isoform Glut4 at physiologic concentrations. AIDS, 16: 859-863. 
22. Hruz, P., H. Murata and H. Qiu et al., 2002. Indinavir induces acute and reversible peripheral insulin resistance in rats. Diabetes, 51: 937-942.

23. Noor, M.A., T. Seneviratne and F.T. Aweeka et al., 2002. Indinavir acutely inhibits insulin-stimulated glucose disposal in humans: a randomized, placebo-controlled study. AIDS, 16: F1-F8.

24. Dube, M.P. and D. Qian and H. EdmondsonMelancon et al., 2002. Prospective, intensive study of metabolic changes associated with 48 weeks of amprenavir-based antiretroviral therapy. Clin. Infect. Dis., 35: 475-481.

25. Noor, M.A., R.A. Parker and E. O'Mara et al., 2004. The effects of HIV protease inhibitors atazanavir and lopinavir/ritonavir on insulin sensitivity in HIV-seronegative healthy adults. AIDS, 18: 2137-2144.

26. Wang, S., R. Mulvey and N. Laing, et al., 2002. Differentiation of atazanavir from other HIVprotease inhibitors in preclinical models of glucose uptake, lipogenesis and proteosome function. Antivir. Ther., 7: L6.

27. Hertel, J., H. Struthers, Horj.C. Baird et al., 2004. A structural basis for the acute effects of HIV protease inhibitors on GLUT4 intrinsic activity. J. Biol. Chem., M410826200.

28. Koster, J.C., M.S. Remedi and H. Qiu et al., 2003. HIV protease inhibitors acutely impair glucosestimulated insulin release. Diabetes, 52: 16951700 .

29. Schutt, M., M. Meier and M.M. Jost et al., 2003. The HIV protease inhibitor indinavir impairs glycogen synthesis in HepG2 hepatoma cells. Exp. Clin. Endocrinol. Diab., 111: 16-20.

30. Schutt, M., J. Zhou and M. Meier et al., 2004. Long-term effects of HIV-1 protease inhibitors on insulin secretion and insulin signaling in INS-1 beta cells. J. Endocrinol., 183: 445-454.

31. Mulligan, K., C. Grunfeld and V.W. Tai et al., 2000. Hyperlipidemia and insulin resistance are induced by protease inhibitors independent of changes in body composition in patients with HIV infection. J. Acquir. Immune. Defic. Syndr., 23: 35-43.

32. Groop, L.C., C. Saloranta and M. Shank et al., 1991. The role of free fatty acid metabolism in the pathogenesis of insulin resistance in obesity and noninsulin-dependent diabetes mellitus. J. Clin. Endocrinol. Metab., 72: 96-107.

33. Unger, R.H., 1995. Lipotoxicity in the pathogenesis of obesity-dependent NIDDM. Genetic and clinical implications. Diabetes, 44: 863-870.
34. Bastard, J.P., M. Caron and H. Vidal et al., 2002. Association between altered expression of adipogenic factor SREBP1 in lipoatrophic adipose tissue from HIV-1-infected patients and abnormal adipocyte differentiation and insulin resistance. Lancet, 359: 1026-1031.

35. Gougeon, M.L., L. Penicaud and B. Fromenty et al., 2004. Adipocytes targets and actors in the pathogenesis of HIV-associated lipodystrophy and metabolic alterations. Antivir. Ther., 9: 161-177.

36. Oral, E.A., V. Simha and E. Ruiz et al., 2002. Leptin-replacement therapy for lipodystrophy. N. Engl. J. Med., 346: 570-578.

37. Riddle, T.M., C.J. Fichtenbaum and D.Y. Hui, 2003. Leptin replacement therapy but not dietary polyunsaturated fatty acid alleviates HIV protease inhibitor-induced dyslipidemia and lipodystrophy in mice. J. Acquir. Immune. Defic. Syndr., 33: 564570 .

38. Mynarcik, D.C., T. Combs and M.A. McNurlan et al., 2002. Adiponectin and leptin levels in HIVinfected subjects with insulin resistance and body fat redistribution. J. Acquir. Immune. Defic. Syndr., 31: 514-520.

39. Grinspoon, S., H. Askari and M. Landt et al., 1997. Effects of fasting and glucose infusion on basal and overnight leptin concentrations in normal-weight women. Am. J. Clin. Nutr., 66: 1352-1356.

40. Mueller, W.M., F.M. Gregoire and K.L. Stanhope et al., 1998. Evidence that glucose metabolism regulates leptin secretion from cultured rat adipocytes. Endocrinology, 139: 551-558.

41. Berg, A.H., T.P. Combs and P.E. Scherer, 2002. ACRP30/adiponectin: an adipokine regulating glucose and lipid metabolism. Trends Endocrinol. Metab., 13: 84-89.

42. Vigouroux, C., M. Maachi and T.H. Nguyen et al., 2003. Serum adipocytokines are related to lipodystrophy and metabolic disorders in HIVinfected men under antiretroviral therapy. AIDS, 17: 1503-1511.

43. Sutinen, J., E. Korsheninnikova and T. Funahashi et al., 2003. Circulating concentration of adiponectin and its expression in subcutaneous adipose tissue in patients with highly active antiretroviral therapy-associated lipodystrophy. J. Clin. Endocrinol. Metab., 88: 1907-1910.

44. Xu, A., S. Yin and L. Wong et al., 2004. Adiponectin ameliorates dyslipidemia induced by the human immunodeficiency virus protease inhibitor ritonavir in mice. Endocrinology, 145: 487-494.

45. Lee, G.A., D.D. Mafong and M.A. Noor et al., 2004. HIV protease inhibitors increase adiponectin levels in HIV-negative men. J. Acquir. Immune. Defic. Syndr., 36: 645-647. 